\title{
Sensor fault detection and diagnosis using zonotopic set-membership estimation
}

\author{
S. Ben Chabane, C. Stoica Maniu, T. Alamo, E.F. Camacho, D. Dumur
}

\begin{abstract}
This paper proposes new sensor fault detection algorithms for linear discrete-time systems with bounded perturbations and bounded measurement noise. This fault detection technique is based on a zonotopic set-membership estimation method. The first proposed fault detection algorithm allows to detect the presence of a fault. A second algorithm leading to guaranteed state estimation in the presence of sensor faults is developed, sometimes leading to conservative results. Thereafter, the last proposed algorithm allows to reduce the conservativeness while offering an estimation of the state closer to the real state of the faulty system. An illustrative example is analyzed to show the performance of the proposed algorithms.
\end{abstract}

Index Terms-Fault detection, set-membership estimation, zonotopes, bounded perturbation, bounded measurement noise.

\section{INTRODUCTION}

Fault Tolerant Control is a relatively new research area that makes possible the development of control laws which allow to maintain current performance close to desirable objectives in the presence of faults. This aims at developing modelbased Fault Detection (FD) algorithms. Usually, the models are represented by adding a fault signal to the faultless model. This fault signal is equal to zero in the fault-free case and it takes non-zero values when a faulty situation occurs. Generally, model-based fault detection algorithms consist in comparing the behaviour of the process and its model when both are fed with the same inputs. Fault detection algorithms check the consistency between measurements and the faultless model.

In the literature, there are two classes of fault detection approaches: active and passive. The active approach is based on generating insensitive residuals with respect to uncertainties but sensitive to faults [1]. The passive approach determines if there is consistency between the model and the measurement [2]. Set-membership estimation approaches [3], [4] are largely used in this class of fault detection methods. Passive fault detection algorithms are applied to test the consistency [5] both in the parameter space [6], [7], [8], and in the state-space [9], [10], [11], [12], [13], [14].

This paper proposes a new passive sensor fault detection algorithm in state-space, based on the zonotopic estimation

\footnotetext{
S. Ben Chabane, C. Stoica Maniu and D. Dumur are with SUPELEC Systems Sciences (E3S) - Automatic Control Department, 3 rue Joliot Curie, F-91192, Gif-surYvette cedex, France (e-mail: \{sofiane.benchabane; cristina.stoica; didier.dumur\} @supelec.fr).

T. Alamo and E.F. Camacho are with Department of Ingeniería de Sistemas y Automática, Universidad de Sevilla, Camino de los Descubrimientos, 41092 Sevilla, Spain (e-mail: alamo@cartuja.us.es, eduardo@esi.us.es).
}

method described in [15]. This estimation method based on the off-line minimization of the $P$-radius of a zonotope has two steps: the prediction step and the correction step. However, applying the zonotopic estimation method [15] in the context of faulty systems does not guarantee the state estimation when a fault has occurred. In order to overcome this situation, a new fault detection algorithm based on the zonotopic state estimation [15] is further proposed. This algorithm leads to guaranteed estimation despite the presence of sensor faults. During the presence of the faults, the idea is to use only the state estimation given by the prediction step of the estimation procedure [15]. In the absence of faults, the two steps (prediction and correction) of the zonotopic set-membership estimation [15] are applied. This procedure leads to guaranteed but conservative estimation. In order to overcome this inconvenient, in the context of faulty systems, a preliminary calibration of the measurements is proposed by centering the measurements relatively to the prediction zonotope. After the measurement calibration, the zonotopic $P$-radius minimization estimation method [15] is applied. This allows to decrease the conservativeness of the proposed estimation technique in a faulty context and decreasing the distance to the real state, when faults are present.

The remainder of the paper is organized as follows. Section II presents useful mathematical notations and basic definitions. Section III formulates the problem of sensor fault detection in the context of systems with bounded disturbances and bounded measurement noise. The estimation method used in the fault-free case is briefly presented in Section IV. Section V details the proposed fault detection algorithms applied to the faulty system and some comments on the sensitivity of these algorithms to detect sensors faults. Section VI proposes an illustrative example which allows to compare the results obtained by the different fault detection algorithms. Finally, some concluding remarks and perspectives are drawn in Section VII.

\section{Mathematical Notations, Definitions And PROPERTIES}

The Minkovsky sum of two sets $\mathcal{A}$ and $\mathcal{B}$ is defined by $\mathcal{A} \oplus \mathcal{B}=\{a+b: a \in \mathcal{A}, b \in \mathcal{B}\}$.

The unitary interval is defined by $\mathbf{B}=[-1,1]$. A unitary box in $\mathbb{R}^{m}$, denoted by $\mathbf{B}^{m}$, is a box composed by $m$ unitary intervals.

A strip is defined as $\mathcal{S}(y, c, \sigma)=\left\{x \in \mathbb{R}^{n}:\left|c^{\top} x-y\right| \leq\right.$ $\sigma\}$.

An $m$-zonotope in $\mathbb{R}^{n}$ is defined by $\mathcal{Z}=\left\{x \in \mathbb{R}^{n}\right.$ : $\left.x=p+H z, z \in \mathbf{B}^{m}\right\}$, where $p \in \mathbb{R}^{n}$ is the center of the 
zonotope, $H \in \mathbb{R}^{n \times m}$ is the shape matrix and $\mathbf{B}^{m}$ is a box (interval vector) composed by $m$ unitary intervals. This is the Minkovsky sum of the $m$ segments defined as $m$ columns of matrix $H$ in $\mathbb{R}^{n}$.

The $P$-radius of an $m$-zonotope $\mathcal{Z}$ is a distance $d(x)=$ $\max _{x \in \mathcal{Z}}\|x-p\|_{P}^{2}$, with $P=P^{\top} \succ 0$.

Given an $m$-zonotope $\mathcal{Z}$ and a vector $c \in \mathbb{R}^{n}$, the zonotope support strip is defined by $\mathcal{F}_{S}=\left\{x \in \mathbb{R}^{n}: q_{l} \leq c^{\top} x \leq q_{u}\right\}$, where the upper bound $q_{u}$ and the lower bound $q_{l}$ satisfy:

$$
\left\{\begin{array}{l}
q_{u}=\max _{x \in \mathcal{Z}} c^{\top} x \\
q_{l}=\min _{x \in \mathcal{Z}} c^{\top} x
\end{array}\right.
$$

and can be calculated as follows:

$$
\begin{aligned}
& q_{u}=c^{\top} p+\left\|H^{\top} c\right\|_{1} \\
& q_{l}=c^{\top} p-\left\|H^{\top} c\right\|_{1}
\end{aligned}
$$

where $\|\cdot\|_{1}$ is the 1-norm of a vector.

Property 1 [16]: The intersection between the zonotope $\mathcal{Z}=p \oplus H \mathbf{B}^{m}$ and the $\operatorname{strip} \mathcal{S}(y, c, \sigma)$ is empty if and only if:

$$
q_{u}<y-\sigma \text { or } q_{l}>y+\sigma .
$$

Property 2 [17]: The Minkovsky sum of two zonotopes $\mathcal{Z}_{1}=p_{1} \oplus H_{1} \mathbf{B}^{m_{1}} \subseteq \mathbb{R}^{n}$ and $\mathcal{Z}_{2}=p_{2} \oplus H_{2} \mathbf{B}^{m_{2}} \subseteq \mathbb{R}^{n}$ is also a zonotope, defined by $\mathcal{Z}=\mathcal{Z}_{1} \oplus \mathcal{Z}_{2}=\left(p_{1}+p_{2}\right) \oplus$ $\left[\begin{array}{ll}H_{1} & H_{2}\end{array}\right] \mathbf{B}^{m_{1}+m_{2}}$.

Property 3 [17]: Given a zonotope $\mathcal{Z}=p \oplus H \mathbf{B}^{m}$ and a matrix $A \in \mathbb{R}^{n \times n}$, then the linear transformation of $\mathcal{Z}$ by the matrix $A$ is $A \mathcal{Z}=(A p) \oplus(A H) \mathbf{B}^{m}$.

Property 4 [18]: Consider a zonotope $\mathcal{Z}=p \oplus H \mathbf{B}^{m}$, a strip $\mathcal{S}(y, c, \sigma)=\left\{x \in \mathbb{R}^{n}:\left|c^{\top} x-y\right| \leq \sigma\right\}$ and the vector $\lambda \in \mathbb{R}^{n}$. Then, a family of zonotopes which is an outer approximation of the intersection between a zonotope $\mathcal{Z}$ and a strip $\mathcal{S}$ is obtained by $\mathcal{Z} \cap \mathcal{S} \subseteq \hat{\mathcal{Z}}(\lambda)=\hat{p}(\lambda) \oplus$ $\hat{H}(\lambda) \mathbf{B}^{m+1}$ such that $\hat{p}(\lambda)=p+\lambda\left(y-c^{\top} p\right)$ and $\hat{H}(\lambda)=$ $\left[\left(I-\lambda c^{\top}\right) H \quad \sigma \lambda\right]$.

\section{PRoblem StATEMENT}

This section focuses on the problem formulation in the case of faulty systems with bounded perturbations and noises.

Consider the following Linear Time Invariant (LTI) discrete-time system with the state and measurement equations:

$$
\left\{\begin{array}{l}
x_{k+1}=A x_{k}+F \omega_{k} \\
y_{k}=c^{\top} x_{k}+\sigma v_{k}+f_{y_{k}}
\end{array}\right.
$$

where $x_{k} \in \mathbb{R}^{n}$ is the state vector, $y_{k} \in \mathbb{R}$ is the measurement, $\omega_{k} \in \mathbb{R}^{n}$ is the state perturbation vector and $v_{k} \in \mathbb{R}$ represents the measurement noise. $A, c, F$ and $\sigma$ have the appropriate dimensions, with the assumption that the pair $\left(c^{\top}, A\right)$ is detectable. It is assumed that the initial state $x_{0}$ belongs to the $m$-zonotope $\mathcal{Z}=p_{0} \oplus H_{0} \mathbf{B}^{m}$ and the perturbations and measurement noise are bounded by compact sets: $\omega_{k} \in \mathbf{B}^{n}$ and $v_{k} \in \mathbf{B}$. The scalar $f_{y_{k}}$ is the sensor fault signal added to the measurement output which is equal to zero in the fault-free case and takes a non-zero value in the presence of faults.

Using the zonotopic set-membership state estimation method presented in [15], the objective is to obtain a state estimation in the presence of abrupt sensors faults.

In this paper, two cases will be considered: fault-free systems and faulty systems.

\section{Zonotopic Set-Membership State Estimation IN THE FAULT-FreE CASE}

This section briefly describes the zonotopic guaranteed state estimation approach [15] for the system (5) in the faultfree case. This estimation method based on the non-increase of the $P$-radius of a zonotope at each iteration allows to manage the trade-off between low computational complexity and accuracy of the estimation. This estimation approach is suitable for the fault-free case. In this approach, it is assumed that $x_{k-1} \in \hat{\mathcal{Z}}_{k-1}=\hat{p}_{k-1} \oplus \hat{H}_{k-1} \mathbf{B}^{r}$, where $\hat{\mathcal{Z}}_{k-1}$ is the zonotopic estimation at time $k-1$. The objective is to compute the zonotope $\hat{\mathcal{Z}}_{k}$ that contains $x_{k}$ at time $k$. To do this, two steps are considered:

1) Prediction step: Using Properties 2 and 3 for system (5), the predicted zonotope $\overline{\mathcal{Z}}_{k}$ is determined as:

$$
\overline{\mathcal{Z}}_{k}=A \hat{p}_{k-1} \oplus\left[\begin{array}{ll}
A \hat{H}_{k-1} & F
\end{array}\right] \mathbf{B}^{r+n} \text {. }
$$

2) Correction step: Using Property 4, the guaranteed state estimation at time $k$ is the outer approximation of the intersection between the predicted state set $\overline{\mathcal{Z}}_{k}$ and the measurement strip $\mathcal{S}$, i.e. $\overline{\mathcal{Z}}_{k} \cap \mathcal{S}\left(y_{k}, c, \sigma\right)$. The strip $\mathcal{S}\left(y_{k}, c, \sigma\right)$ is obtained using the output measurement at time $k$ according to the following equation:

$$
\mathcal{S}\left(y_{k}, c, \sigma\right)=\left\{x \in \mathbb{R}^{n}:\left|c^{\top} x-y_{k}\right| \leq \sigma\right\}
$$

This outer approximation is parametrized by the vector $\lambda \in$ $\mathbb{R}^{n}$, leading to the following family of zonotopes [15]:

$$
\hat{\mathcal{Z}}_{k}(\lambda)=\hat{p}_{k}(\lambda) \oplus \hat{H}_{k}(\lambda) \mathbf{B}^{r+n+1}
$$

with

$$
\left\{\begin{array}{l}
\hat{p}_{k}(\lambda)=A \hat{p}_{k-1}+\lambda\left(y_{k}-c^{\top} A \hat{p}_{k-1}\right) \\
\hat{H}_{k}(\lambda)=\left[\begin{array}{lll}
\left(I-\lambda c^{\top}\right) A \hat{H}_{k-1} & \left(I-\lambda c^{\top}\right) F & \sigma \lambda
\end{array}\right]
\end{array}\right.
$$

Using the zonotope reduction property [17], it is possible to set the zonotope complexity of $\hat{\mathcal{Z}}_{k}(\lambda)$ by imposing the number of its segments.

The vector $\lambda$ is then determined in order to minimize the $P$-radius of the zonotope $\hat{\mathcal{Z}}_{k}$ at each sample time. The solution (see [15] for more details) is found by minimizing the scalar $\beta \in(0,1)$ using the bisection algorithm and offline solving the following Linear Matrix Inequality (LMI) optimization problem:

$$
\max _{\tau, P, \beta, Y} \tau
$$


subject to

$$
\left\{\begin{array}{l}
0 \leq \beta<1 \\
\frac{(1-\beta) P}{\sigma^{2}+\text { const }} \succeq \tau I \\
{\left[\begin{array}{cccc}
\beta P & 0 & 0 & A^{\top} P-A c Y^{\top} \\
* & F^{\top} F & 0 & F^{\top} P-F^{\top} c Y^{\top} \\
* & * & \sigma^{2} & Y^{\top} \sigma \\
* & * & * & P
\end{array}\right] \succeq 0}
\end{array}\right.
$$

with const $=\max _{\omega \in \mathbf{B}^{n}}\|F \omega\|_{2}^{2}$ and $Y=P \lambda$.

This method offers good accuracy of the estimation bounds with small computation time. It allows to estimate the state of the system (5) in the fault-free case. The objective is to use a similar estimation method to detect the fault when it has occurred and try to obtain a good estimation despite the presence of sensor faults.

\section{Fault Detection Based on Zonotopic SET-Membership State Estimation}

Model-based fault detection of dynamic processes is based on the use of the model (state equation) to check the consistency of the observed behaviour (measurement equation). In the case of the model (5), the idea is to check the consistency between the prediction zonotope $\left(\overline{\mathcal{Z}}_{k}\right)$ and the set of measurements $\mathcal{S}\left(y_{k}, c, \sigma\right)$. Using the definition of the zonotope support strip and Property 1, a fault has occurred if the set $\overline{\mathcal{Z}}_{k} \cap \mathcal{S}\left(y_{k}, c, \sigma\right)$ is empty. In other words, a fault has occurred if the equation (4) holds. In the fault-free case, the zonotopic estimation method [15] is used.

Algorithm 1 provides a general conceptual form of the fault detection strategy based on checking consistency between the model and the measurement. The idea of this algorithm is the following. At each time instant $k$ the prediction zonotope $\overline{\mathcal{Z}}_{k}$ is built according to (6). Using the output measurement obtained from the sensor, the strip $\mathcal{S}\left(y_{k}, c, \sigma\right)$ is also built according to (7). Then, the consistency between the predicted zonotopic state estimation and the measurement strip is checked. If the consistency is proved, the $P$-radius minimization method is used to compute the intersection between the predicted zonotopic state estimation and the measurement strip according to (8), (9) and (10). Otherwise, if the intersection between the predicted zonotopic state estimation $\overline{\mathcal{Z}}_{k}$ and the measurement strip is empty according to (4), a fault is considered to be present.

\footnotetext{
Algorithm 1. FD using consistency test

1. $k \leftarrow 0$

2. $\mathcal{Z}_{0} \leftarrow p_{0} \oplus H_{0} \mathbf{B}^{m}$

3. for $k=1: N$

4. Compute the predicted zonotope $\overline{\mathcal{Z}}_{k}$ according to the equation (6). Use the output measurement $y_{k}$, i.e. the strip $\mathcal{S}\left(y_{k}, c, \sigma\right)$ according to (7).

5. if $\quad \overline{\mathcal{Z}}_{k} \cap \mathcal{S}\left(y_{k}, c, \sigma\right)=\emptyset$

$6 . \quad$ Indicate fault.

7. else

8. $\quad$ Compute the zonotope $\hat{\mathcal{Z}}_{k}$ that fulfills $\overline{\mathcal{Z}}_{k} \cap \mathcal{S}\left(y_{k}, c, \sigma\right)$ according to equations
}
9. end if
10. $\quad k \leftarrow k+1$
11. end for.

(8), (9) and (10)

Here $N$ is the length of the simulation time, $p_{0}$ and $H_{0}$ denote the initial state zonotope (which is sufficiently large).

The objective is now to find a solution to estimate the state of the system when the fault has occurred in the step 6 of Algorithm 1. Two solutions will be further discussed.

\section{A. Fault Detection using only the prediction step}

The goal is to obtain a guaranteed estimation in the presence of a fault. The idea is to improve Algorithm 1 when a fault has occurred. It consists in using just the prediction zonotope if an inconsistency is detected. In the faulty situation, the measurement strip is not considered and the estimation zonotope will not be changed at the correction step. After the sensor recovery, both the prediction and correction steps are used in order to estimate the state, using equations (8), (9) and (10). Algorithm 2 presents this idea.

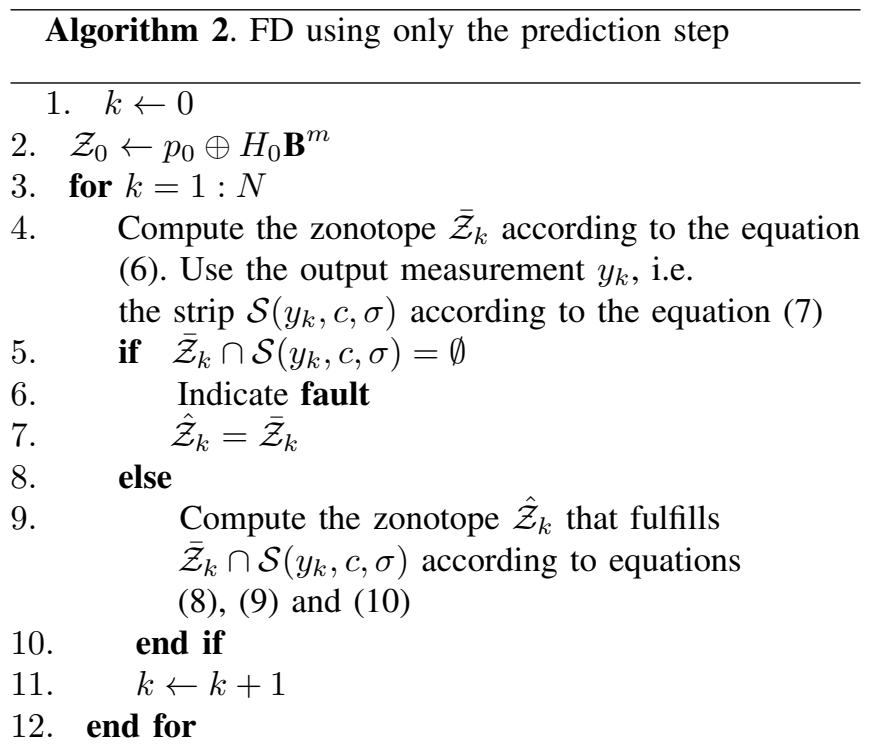

This gives a guaranteed state estimation but the estimation accuracy could deteriorate.

In order to increase accuracy, a calibration of the measurement is proposed in the next subsection.

\section{B. Calibration of the measurement strip}

The goal is to get a good estimation which is closer to the real state when a fault is detected. It consists in shifting the measurement strip $\mathcal{S}\left(y_{k}, c, \sigma\right)$ (e.g. the blue strip in Fig. 1) to the center of the predicted zonotope $\overline{\mathcal{Z}}_{k}$ (e.g. the red strip in Fig. 1). Assume that $q_{u}<y_{k}-\sigma$ (see the blue strip in Fig. 1) according to the condition of inconsistency (4). The idea is to calibrate the output measurement $y_{k}$ as follows:

$$
y_{k} \leftarrow y_{k}-d_{1}-\frac{d_{2}}{2}-\sigma=\frac{q_{u}+q_{l}}{2}
$$


where $d_{1}=y_{k}-q_{u}-\sigma$ and $d_{2}=q_{u}-q_{l}$. If the condition of inconsistency $q_{l}>y_{k}+\sigma$ (see the green strip in Fig. 1) holds in (4), the output measurement is calibrated as follows:

$$
y_{k} \leftarrow y_{k}+d_{3}+\frac{d_{2}}{2}+\sigma=\frac{q_{u}+q_{l}}{2}
$$

where $d_{3}=q_{l}-y_{k}-\sigma$.

The new obtained strip is the dotted red strip (see Fig. 1). After this calibration, the zonotopic state estimation is computed using the $P$-radius minimization approach presented in Section IV. When there is no fault, the method of state estimation based on the $P$-radius minimization method presented in Section IV is applied. In a faulty situation, Algorithm 3 provides a solution.

\section{Algorithm 3. FD with measurement calibration}

1. $k \leftarrow 0$

2. $\mathcal{Z}_{0} \leftarrow p_{0} \oplus H_{0} \mathbf{B}^{m}$

3. for $k=1: N$

4. Compute the zonotope $\overline{\mathcal{Z}}_{k}$ according to the equation (6). Use the output measurement $y_{k}$, i.e. the strip $\mathcal{S}\left(y_{k}, c, \sigma\right)$ according to the equation (7)

5. $\quad$ if $\quad \overline{\mathcal{Z}}_{k} \cap \mathcal{S}\left(y_{k}, c, \sigma\right)=\emptyset$

6. Indicate fault

7. if $\left(q_{u}<y_{k}-\sigma\right)$ or $\left(q_{l}>y_{k}+\sigma\right)$

8. $\quad y_{k} \leftarrow \frac{q_{u}+q_{l}}{2}$

9 end if

10. end if

11. Compute the zonotope $\hat{\mathcal{Z}}_{k}$ that fulfills $\overline{\mathcal{Z}}_{k} \cap \mathcal{S}\left(y_{k}, c, \sigma\right)$ according to (8), (9) and (10)

12. $k \leftarrow k+1$

13. end for

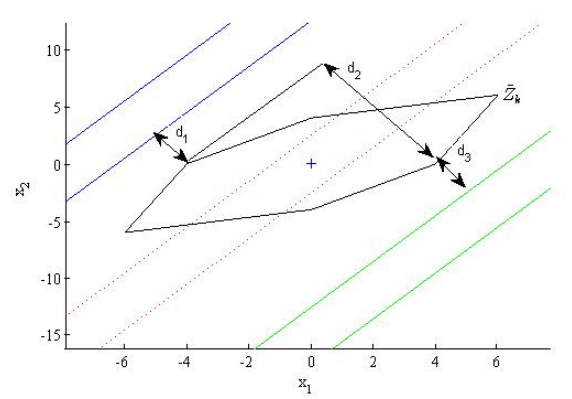

Fig. 1. Measurement calibration

Consider that the real state estimation $x_{k}$ belongs to the prediction zonotope $\overline{\mathcal{Z}}_{k}$ but it is outside the measurement strip (i.e. the dotted red strip in Fig. 1). In this case, Algorithm 3 does not guarantee the state estimation, but it still offers an estimation closer to the real state than the one obtained using Algorithm 2. This can be suitable for control perspectives.

\section{Fault sensitivity of the proposed algorithms}

Sensitivity toward faults is an important charateristic of fault detection algorithms. The minimum abrupt fault that will be detected by the proposed algorithms for the output sensor fault $f_{y_{k}}$ has to be determined.

The two cases of inconsistency (see equation (4)) are considered. The first case is when the following equation holds:

$$
q_{u}<y_{k}-\sigma \text {. }
$$

Substituting $q_{u}$ from (2) and $y_{k}$ from (5), the following equation is obtained:

$$
c^{\top} \hat{p}_{k}+\left\|\hat{H}_{k}^{\top} c\right\|_{1} \leq c^{\top} x_{k}+\sigma v_{k}+f_{y_{k}}-\sigma .
$$

Assuming the worst-case with positive transition in $f_{y_{k}}$ of $x_{k} \in \hat{\mathcal{Z}}_{k}(\lambda)$ and $v_{k} \in \mathbf{B}$ by minimizing $c^{\top} x_{k}$, i.e. using (3), and minimizing $\sigma v_{k}$, i.e. $v_{k}=-1$, the condition of the magnitude of $f_{y_{k}}$ is obtained as:

$$
c^{\top} \hat{p}_{k}+\left\|\hat{H}_{k}^{\top} c\right\|_{1} \leq c^{\top} \hat{p}_{k}-\left\|\hat{H}_{k}^{\top} c\right\|_{1}-\sigma+f_{y_{k}}-\sigma,
$$

which is equivalent to:

$$
f_{y_{k}} \geq 2 \sigma+2\left\|\hat{H}_{k}^{\top} c\right\|_{1} .
$$

The condition (16) means that the sensor fault is detected when its positive transition is greater than $2 \sigma+2\left\|H^{\top} c\right\|_{1}$. The second case of inconsistency occurs when the following equation holds:

$$
q_{l}>y_{k}+\sigma
$$

Substituting $q_{u}$ from (3) and $y_{k}$ from (5), the following equation is obtained:

$$
c^{\top} \hat{p}_{k}-\left\|\hat{H}_{k}^{\top} c\right\|_{1} \geq c^{\top} x_{k}+\sigma v_{k}+f_{y_{k}}+\sigma .
$$

Assuming the worst-case with negative transition in $f_{y_{k}}$ of $x_{k} \in \hat{\mathcal{Z}}_{k}(\lambda)$ and $v_{k} \in \mathbf{B}$ by maximizing $c^{\top} x_{k}$, i.e. using (2), and maximizing $\sigma v_{k}$, i.e. $v_{k}=1$, the condition of the magnitude of $f_{y_{k}}$ is obtained as:

$$
c^{\top} \hat{p}_{k}-\left\|\hat{H}_{k}^{\top} c\right\|_{1} \geq c^{\top} \hat{p}_{k}+\left\|\hat{H}_{k}^{\top} c\right\|_{1}+\sigma+f_{y_{k}}+\sigma,
$$

which is equivalent to:

$$
f_{y_{k}} \leq-2 \sigma-2\left\|\hat{H}_{k}^{\top} c\right\|_{1} .
$$

The condition (20) means that the sensor fault is detected when its negative transition is smaller than $2 \sigma+2\left\|H^{\top} c\right\|_{1}$.

Summarizing, the abrupt sensor fault is detected when:

$$
\left|f_{y_{k}}\right| \geq 2 \sigma+2\left\|\hat{H}_{k}^{\top} c\right\|_{1} \text {. }
$$

An example where the abrupt fault is taken equal to $2 \sigma$ is considered in Fig. 2. Here the fault is not detected, i.e. the intersection between the predicted black 20-zonotope and the measurement strip (in blue) is not empty in spite of the fact that the real state is outside the measurement strip. This figure illustrates that the red zonotope computed using the $P$-radius-based estimation method can contain the real state and in the worst-case it will be closer to the real state. Improving the sensitivity of the fault detection algorithm will be addressed in future work.

In the next section, an illustrative example showing the performance of Algorithms 2 and 3 is presented. 


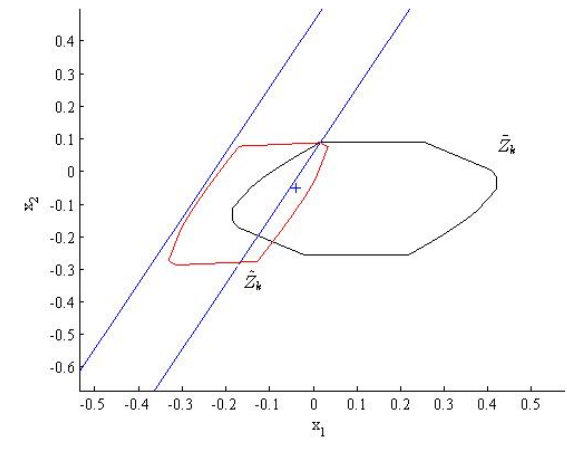

Fig. 2. Fault detection sensitivity

\section{ILLUSTRATIVE EXAMPLE}

Consider the following LTI discrete-time system:

$$
\left\{\begin{array}{l}
x_{k+1}=\left[\begin{array}{cc}
0.8 & 0.2 \\
-0.5 & 0.8
\end{array}\right] x_{k}+\left[\begin{array}{c}
-0.12 \\
0.02
\end{array}\right] \omega_{k} \\
y_{k}=\left[\begin{array}{ll}
-2 & 1
\end{array}\right] x_{k}+0.2 v_{k}+f_{y_{k}}
\end{array}\right.
$$

with $\left\|v_{k}\right\|_{\infty} \leq 1,\left\|\omega_{k}\right\|_{\infty} \leq 1$. The initial state belongs to the box $3 \mathbf{B}^{2}$. The order of the $m$-zonotope is limited to $m \leq 20$ (number of its segments) in order to have a fast simulation. The sensor fault $f_{y_{k}}$ is introduced between sample times $k=30$ and $k=40$. In this example, the results obtained by the $P$-radius minimization method without fault detection (detailed in Section IV), and with fault detection (Algorithm 2 and Algorithm 3) are analyzed.

Figures 3 and 4 show the bounds of $x_{1}$ and $x_{2}$ obtained by the $P$-radius minimization method (blue lines). The red stars represent the real state of the system. These points are not between the upper bound and the lower bound of the state estimation when the sensor fault $f_{y_{k}}$ is present.

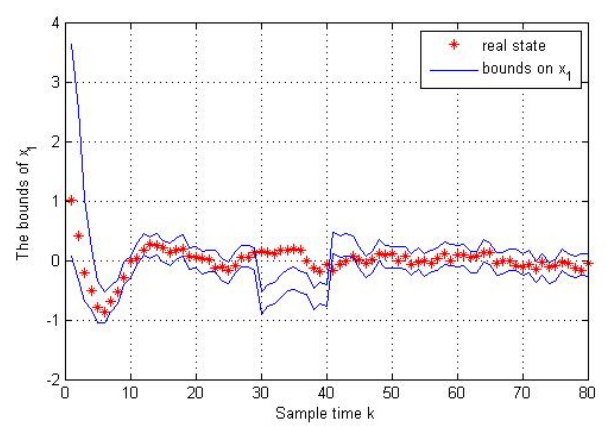

Fig. 3. Bounds on $x_{1}$ using the $P$-radius minimization method

Figure 5 shows the detection of the fault during the estimation via Algorithm 2. The fault occurred when the signal is equal to 1 and equal to 0 otherwise. Figures 6 and 7 show the bounds of $x_{k}$ obtained by Algorithm 2 (blue lines). The red stars represent the real state of the system. These points are between the upper and lower bounds of $x_{k}$. The reader can observe that when the fault is present $(k=30$ to $k=40$ ), the estimated bounds are increased. This is due to

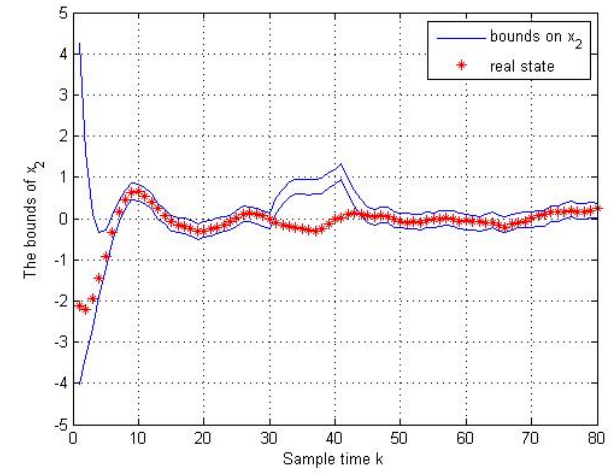

Fig. 4. Bounds on $x_{2}$ using the $P$-radius minimization method

the omission of the correction step in the estimation method (i.e. only the prediction step is used in Algorithm 2).

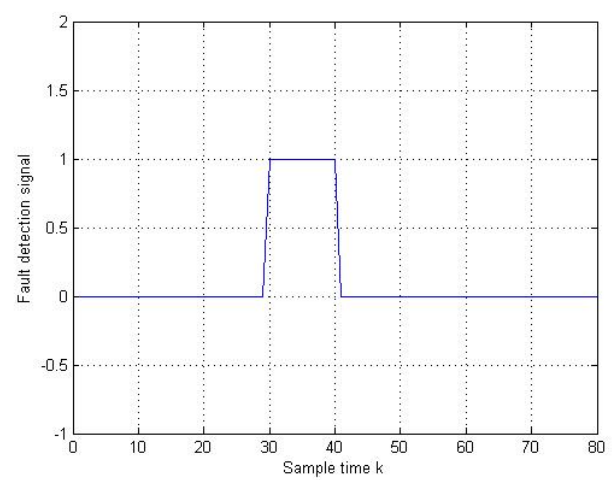

Fig. 5. Fault detection signal using Algorithm 2 (step 6)

The result of fault detection via Algorithm 3 is the same as the result obtained by Algorithm 2 as shown in Fig. 5 .

Figures 8 and 9 show the bounds of $x_{k}$ obtained via Algorithm 3 (blue lines). The red stars represent the real state of the system. The distance between the upper and lower bounds obtained by Algorithm 3 (Figures 8, 9) is less than the distance between the upper and lower bounds obtained by Algorithm 2 (Figures 6,7). Even if Algorithm 3 does not guarantee the state estimation bounds in presence of faults, the results can be less conservative compared to Algorithm 2. This could make Algorithm 3 more suitable for Fault Tolerant Control perspectives than Algorithm 2.

\section{CONCLUSION}

In this paper, two fault detection algorithms based on consistency test have been proposed. These algorithms are based on the $P$-radius minimization method of the zonotopic estimation. The first approach (Algorithm 2) gives a guaranteed but conservative estimation in the presence of sensor faults. The second approach (Algorithm 3) gives an estimation method with reduced conservativeness when faults occur. This algorithm is better than Algorithm 2 to control perspectives. An example illustrates the performance of the two algorithms. 


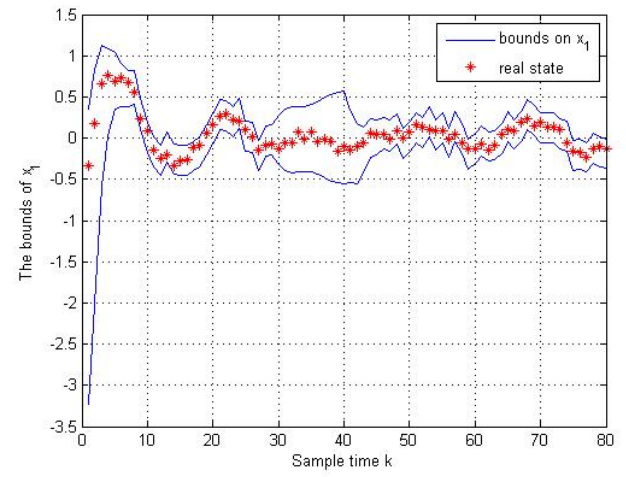

Fig. 6. Bounds on $x_{1}$ using Algorithm 2

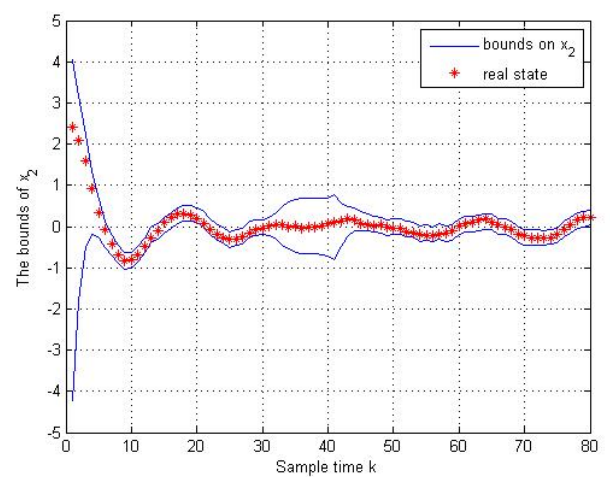

Fig. 7. Bounds on $x_{2}$ using Algorithm 2

Future work will focus on extending this Fault Detection method to the case of multivariable systems with interval uncertainties and to apply these algorithms to Fault Tolerant Control purposes. Considering incipient faults will be also investigated in future developments.

\section{REFERENCES}

[1] J. Chen and R. Patton, Robust model-based Fault Diagnosis for dynamic systems. Kluwer Academic Publishers, 1999.

[2] V. Puig, J. Quevedo, and T. Escobet, "Robust fault detection approaches using interval models," in Proc. on IFAC World Congress, Spain, 2002.

[3] F. C. Schweppe, "Recursive state estimation: unknown but bounded errors and system inputs," IEEE Trans. Automat. Contr., vol. 13(1), pp. 22-28, 1968.

[4] B. T. Polyak, S. A. Nazin, C. Durieu, and E. Walter, "Ellipsoidal parameter or state estimation under model uncertainty," Automatica, vol. 40, pp. 1171-1179, 2004.

[5] V. Puig, "Fault Diagnosis and Fault Tolerant Control using setmembership approaches: Application to real case studies," International Journal of Applied Mathematics and Computer Science, vol. 20(4), pp. 619-635, 2010.

[6] A. Ingimundarson, J. M. Bravo, V. Puig, T. Alamo, and P. Guerra, "Robust fault detection using zonotope-based set-membership consistency test," International Journal of Adaptive Control and Signal Processing, vol. 23(4), pp. 311-330, 2008.

[7] A. Ingimundarson, J. Bravo, V. puig, and T. Alamo, "Robust fault diagnosis using parrallelotope-based set-membership consistency tests," in Proc. of European Control Conference, Seville, Spain, 2005.

[8] J. Blesa, V. Puig, and J. Saludes, "Robust fault detection using polytope-based set-membership consistency test," in Conference on Control and Fault Tolerant Systems, Nice, France, 2010.

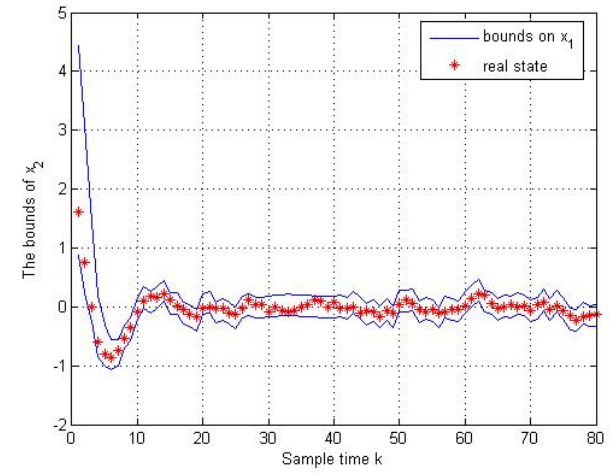

Fig. 8. Bounds on $x_{1}$ using Algorithm 3

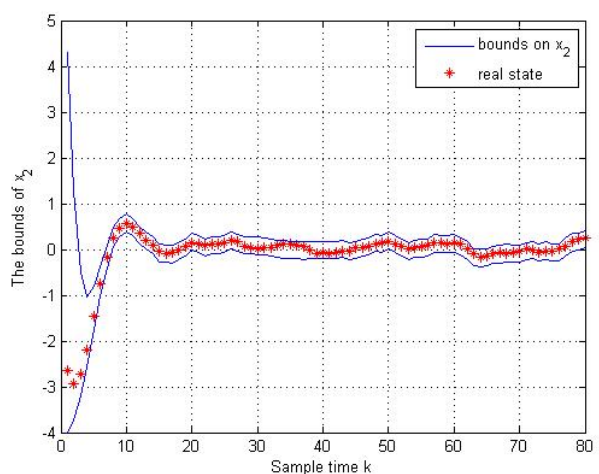

Fig. 9. Bounds on $x_{2}$ using Algorithm 3

[9] P. Planchon and J. Lunze, "Diagnosis of linear systems with structured uncertainties based on guaranteed state observation," International Journal of Control, Automation and Systems, vol. 6(3), pp. 306-319, 2008.

[10] S. M. Tabatabacipour, P. F. Odgaard, and T. Bak, "Fault detection of a benchmark wind turbine using interval analysis," in American Control Conference, 2012, pp. 4387-4392, Montréal, Canada.

[11] F. Nejjari, V. Puig, S. M. Ora, and A. Sadeghzadeh, "Robust fault detection for LPV systems using interval observers and zonotopes,' in Proc. of the 48th IEEE Conference on Decision and Control, 2009 pp. 1002-1007, Shanghai, P.R. China.

[12] M. M. Seron and J. A. Doná, "Robust actuator fault compensation accounting for uncertainty in the fault estimation," International Journal of Adaptive Control and Signal Processing, vol. DOI: 10.1002/acs.2453, 2013.

[13] S. Olaru, J.A. De Doná, M. Seron, and F. Stoican, "Positive invariant sets for fault tolerant multisensor control schemes," International Journal of Control, vol. 83, no. 12, pp. 2622-2640, 2010.

[14] M. M. Seron, J. A. De Doná, and S. Olaru, "Fault tolerant control allowing sensor healthy-to-faulty and faulty-to-healthy transitions," Automatic Control, IEEE Transactions on, vol. 57, no. 7, pp. 1657 1669, 2012.

[15] V. T. H. Le, C. Stoica, T. Alamo, E. F. Camacho, and D. Dumur, "Zonotopic guaranteed state estimation for uncertain systems," Automatica, vol. 49(1), pp. 3418-3424, 2013.

[16] A. Vicino and G. Zappa, "Sequential approximation of feasible parameter sets for identification with set membership uncertainty," IEEE Trans. Automat. Contr., vol. 41, pp. 774-785, 1996.

[17] C. Combastel, "A state bounding observer based on zonotopes," in Proc. of European Control Conference, Cambridge, UK, 2003.

[18] T. Alamo, J. M. Bravo, and E. F. Camacho, "Guaranteed state estimation by zonotopes," Automatica, vol. 41, pp. 1035-1043, 2005. 